\title{
Promoting Safe Prescribing in Primary Care With a Contraceptive Vital Sign: A Cluster- Randomized Controlled Trial
}

Eleanor Bimla Schwarz, MD, MS

Sara M. Parisi, MS, MPH

Sanitbia L. Williams, BS

Grant J. Shevchik, MD

Rachel Hess, MD, MS

University of Pittsburgh, Pittsburgh, Pennsylvania
Conflicts of interest: authors report none.

\section{CORRESPONDING AUTHOR}

Eleanor Bimla Schwarz, MD, MS

Department of Medicine

University of Pittsburgh

$230 \mathrm{McKee} \mathrm{Pl}$, Ste 600

Pittsburgh, PA 15213

schwarzeb@upmc.edu

\begin{abstract}
PURPOSE Routine assessment of women's pregnancy intentions and contraceptive use-a so-called contraceptive vital sign-may help primary care physicians identify patients who need preconception or contraceptive counseling and be of particular benefit when teratogenic medications are prescribed.
\end{abstract}

METHODS We conducted a cluster-randomized controlled trial to evaluate the effect of a contraceptive vital sign on primary care documentation of contraceptive use and change in primary care physicians' provision of family planning services. Academic internists in the intervention group $(n=26)$ were provided with information on their female patients' pregnancy intentions and contraceptive use immediately before visits; internists in the control group $(n=27)$ received only standard intake information. Data were abstracted from the electronic health record for 5,371 visits by 2,304 women aged 18 to 50 years.

RESULTS Documentation of contraception increased from baseline, from 23\% to $57 \%$ in the intervention group, but remained $28 \%$ in the control group, a change of +77.4 (95\% confidence interval [Cl], 70.7 to 84.1 ) adjusted percentage points in the former vs $+3.1(95 \% \mathrm{Cl}, 1.2$ to 5.0$)$ in the latter $(P<.001)$. For visits involving a teratogenic prescription, documentation increased from $14 \%$ to $48 \%$ in the intervention group and decreased from $29 \%$ to $26 \%$ in the control group, a change of $+61.5(95 \% \mathrm{Cl}, 35.8$ to 87.1$)$ adjusted percentage points in the former vs $-0.3(95 \% \mathrm{Cl},-4.3$ to 3.6$)$ in the latter $(P<.001)$. Provision of new family planning services increased only minimally with this intervention, however. When women with documented nonuse of contraception were prescribed potential teratogens, only $7 \%$ were provided family planning services.

CONCLUSIONS A contraceptive vital sign improves documentation of contraceptive use; however, ongoing efforts are needed to improve provision of preconception and contraceptive services.

Ann Fam Med 2012;10:516-522. doi:10.1370/afm.1404.

\section{INTRODUCTION}

W

omen whose medical condition or medication use may increase the risk of adverse pregnancy outcomes need preconception guidance and contraceptive counseling to reduce the risk of unintended pregnancy and optimize pregnancy timing. One-half of US pregnancies remain unintended, ${ }_{1}^{1}$ however, and family planning services are provided during only $5 \%$ to $20 \%$ of visits made by women prescribed potentially teratogenic medications. ${ }^{2}$ As a result, approximately $6 \%$ of US pregnancies are exposed to potentially teratogenic class $\mathrm{D}$ or $\mathrm{X}$ medications. ${ }^{3}$

As the majority of potentially teratogenic medications are prescribed by primary care physicians, ${ }^{2,4}$ there is a particular need for these physicians to provide preconception and contraceptive counseling. Primary care physicians have reported that one barrier to safe prescribing to women of 
reproductive age is difficulty ascertaining their pregnancy intentions, and they have expressed interest in strategies for routinely assessing chance of pregnancy. ${ }^{5,6}$ Obtaining a so-called contraceptive vital sign, similar to efforts to assess pain as a vital sign, ${ }^{7,8}$ could help prompt preconception counseling.

In this study, we evaluated the feasibility and efficacy of this type of routine intake assessment of women's pregnancy intentions and contraceptive use on the documentation of contraception and primary care physicians' provision of family planning services. We hypothesized that a contraceptive vital sign would increase documentation of contraception and increase provision of family planning services, especially for women prescribed potentially teratogenic medications.

\section{METHODS}

\section{Setting and Participants}

We conducted a cluster-randomized trial between October 2008 and April 2010 to evaluate the introduction of a contraceptive vital sign in a large, academic general internal medicine practice that has collected patiententered intake information (eg, alcohol use, physical activity, last menstrual period) using wirelessly networked tablet computers since 2004. These computers use branched logic to select appropriate intake questions for each patient. The computer synthesizes and formats the information into a paper report for physicians., ${ }^{9,10}$ All primary care physicians at this practice were invited to participate. As this practice is a residency training site, participating physicians were both residents and supervising physicians. This study was approved by the University of Pittsburgh Institutional Review Board.

\section{Intervention Design}

Physicians were randomized to an intervention group or control group. When female patients aged 18 to 50 years were scheduled to see intervention physicians, a pair of questions asking about pregnancy intentions and recent contraceptive use - the contraceptive vital sign-were added to their intake questionnaire. Specifically, they were asked "Are you currently pregnant or trying to become pregnant?" and provided with 6 response options: "no," "not trying to get pregnant but wouldn't mind being pregnant," "trying to get pregnant," "currently pregnant," "been through menopause," and "prefer not to answer." Women who responded either "no" or "not trying to get pregnant..." were then asked: "There are many ways that people try to avoid becoming pregnant. Which are you using?" and were provided with a list of contraceptive methods. Response options included "no method of birth control," "not sexually active with men in the past 3 months," and "prefer not to answer." If a patient reported that she was pregnant, trying to become pregnant, wouldn't mind becoming pregnant, or trying to avoid pregnancy but not using any contraception, the intake report provided to physicians included the warning statement "Consider chance of pregnancy when prescribing" in bold text, unless the woman responded that she had not had sex within the past 3 months. The decision to consider women abstinent for 3 months as at low risk of pregnancy reflected the majority opinion of this practice's clinicians. Patients previously indicating that they were menopausal or had undergone hysterectomy or tubal ligation were not asked the questions. Also, because of a programming error, women making annual preventive visits were not asked the questions.

Patients seeing control physicians were asked only the standard intake questions. This study was conducted in conjunction with the introduction of clinical decision support integrated into the clinic's electronic health record (EHR), which alerted physicians when they initiated a prescription for a potentially teratogenic medication.

\section{Data Collection}

We reviewed deidentified EHR data from all visits by women aged 18 to 50 years who started to complete a computerized intake questionnaire during a baseline period (May 2007-September 2008) and the intervention period (October 2008-April 2010). Abstracted data included evidence of new contraceptive prescriptions; referrals for placement of an intrauterine contraceptive, contraceptive implant, or diaphragm, contraceptive counseling received within the past 3 months; prior contraceptive prescriptions or devices that were still active; pregnancy tests; potentially teratogenic medications prescribed at the visit; type of clinical encounter (new vs return, usual physician vs other); and patients' age, race, and marital status. Responses to the contraceptive vital sign questions were extracted from the tablet computers and linked to EHR data by an honest broker. We eliminated all visits with evidence of sterilization, menopause, or infertility, as well as all annual preventive visits, which did not receive the intervention as planned. Additionally, because the intake system provides physicians with information patients have given at previous visits, in a small number of cases, control physicians inadvertently received contraceptive vital sign information from previous visits with intervention physicians; we also eliminated these "crossover" visits.

\section{Statistical Analysis}

Intervention and control physicians were compared using $\chi^{2}$ tests and Wilcoxon rank sum tests, and the characteristics of visits made to the 2 groups during 
the baseline period were compared using mixed-effects models with random effects to address clustering by physician and patient. We calculated the response rate and range of responses to each of the contraceptive vital sign questions. We calculated the proportion of visits in each study group with documentation of contraception, overall and for the subgroup of visits involving the prescription of a potential teratogen, at baseline and during the intervention.

To test whether there was significantly different change in documentation in the intervention group, we constructed visit-level generalized linear mixed-effects models with a repeated time measure adjusting for visit characteristics including patient characteristics (aged $\geq 30$ years, married, white), and physician characteristics (sex, resident, receipt of clinical decision support when prescribing a potential teratogen). The models contained an interaction term to test whether changes over time differed significantly between groups. ${ }^{11}$ Using nearly identical models, we also investigated whether the contraceptive vital sign affected the rate of prescription of potentially teratogenic medications and provision of new family planning services (ie, new contraception prescriptions, contraceptive counseling, pregnancy testing, or referral to a family planning specialist). Models predicting documentation of contraception included a random effect for patients only, as this information was collected before seeing the physician. Models predicting physician prescribing and counseling behavior included a random effect for physician only, as models containing crossed random effects for physicians and patients would not converge. We also tested models adjusting only for patient clustering, but the difference between the models was not appreciable.

From each model, we estimated the adjusted absolute change in the outcome over time and the $95 \%$ confidence interval (CI) (holding covariates at their grand means). Finally, we calculated the residual intraclass correlation coefficient (ICC) for provision of teratogenic prescriptions and family planning services. ${ }^{12}$

\section{Community-Based Extension}

As an extension of this trial, we pilot tested the use of a similar intake system in a community-based family practice. Given the smaller number of primary care physicians in this practice $(\mathrm{N}=13)$, implementation was not randomized by physician; rather, the front desk staff randomly requested that $10 \%$ of women use the new intake system. We therefore compared rates of potentially teratogenic prescriptions among women who were and were not asked to provide the contraceptive vital sign and investigated whether provision of new family planning services when potential teratogens were prescribed was higher among visits involving the contraceptive vital sign.

All analyses were conducted using Stata version 11 IC (StataCorp LP), P values less than .05 were considered significant.

\section{RESULTS}

Figure 1 shows the flow of participating primary care physicians and visits. We abstracted data from 11,621 visits. We eliminated 1,834 visits with women who
Figure 1. Flow diagram describing physician clusters and patient encounters from the time of recruitment to analysis.

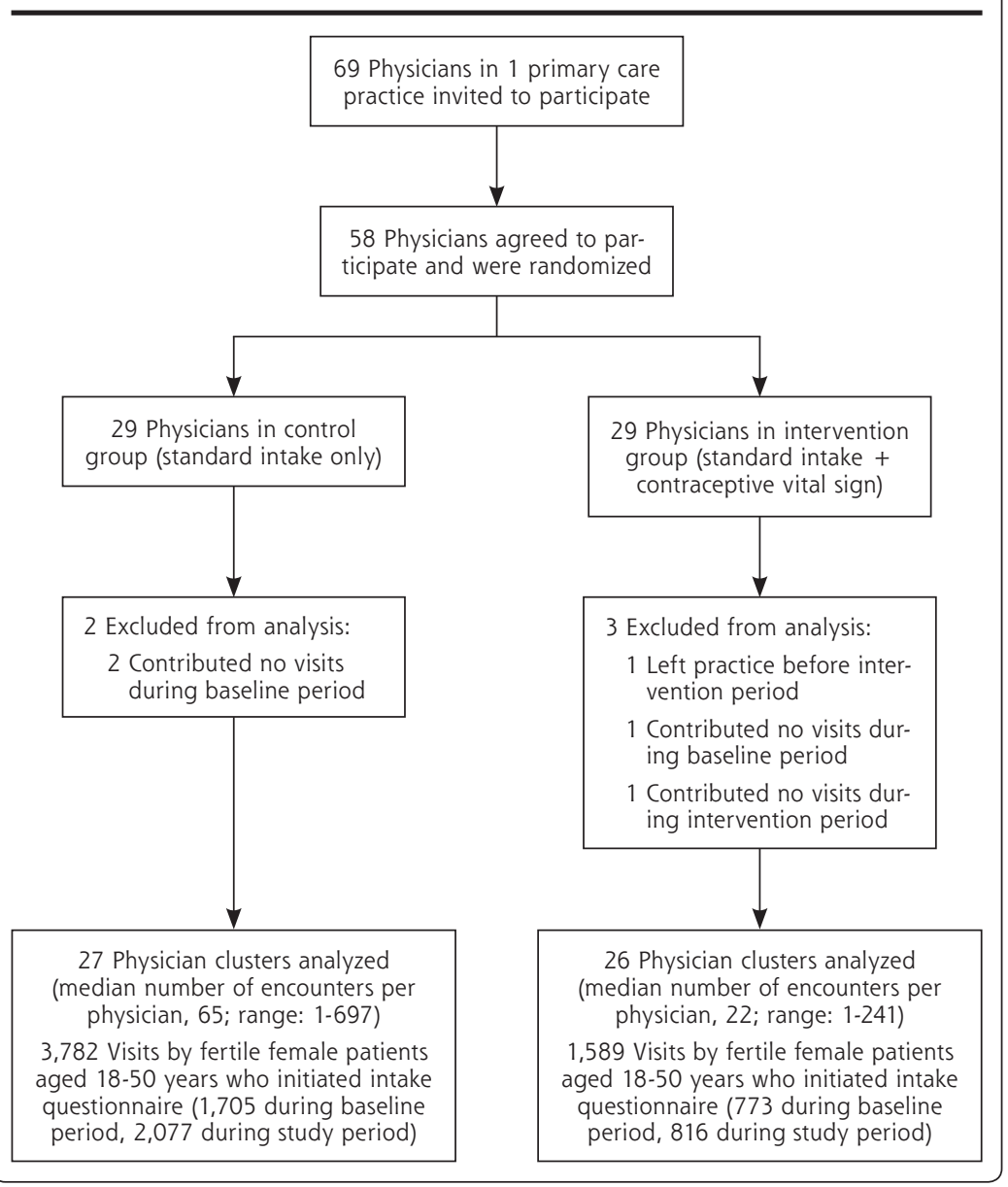


had evidence of sterilization, menopause, or infertility (16\% of all visits; $22 \%$ of visits with a potentially teratogenic prescription). We also eliminated 158 visits in which a control physician inadvertently received contraceptive vital sign information entered on a previous visit and 4,258 annual preventive visits, leaving 5,371 visits made by 2,304 unique women. There were no significant differences in physician characteristics between groups (Table 1).

\section{Feasibility and Acceptability of the Intervention}

During the 816 visits in which women were asked the contraceptive vital sign questions, $93 \%$ provided answers. The remaining $7 \%$ either skipped the question or did not finish the questionnaire. In total, intervention physicians were notified to "consider chance of pregnancy when prescribing" in $13.5 \%$ of visits (110 of 816 ) in which contraceptive vital sign data were collected. Responses to the contraceptive vital sign questions are given in Table 2.

\section{Efficacy of the Intervention}

Figure 2 displays the proportion of visits with documented use of contraception before and during the intervention period, for all visits as well as the subgroup of visits with a potentially teratogenic prescription. There was significantly greater improvement in documentation in the intervention group compared with the control group: +77.4 (95\% CI, 70.7 to 84.1 ) adjusted percentage points in the former vs +3.1 (95\% CI, 1.2 to 5.0$)$ in the latter $(P<.001)$. A similar increase was seen among visits with a teratogenic prescription: +61.5 (95\% CI, 35.8 to 87.1 ) adjusted percentage points vs $-0.3(95 \%$ $\mathrm{CI},-4.3$ to $\left.3.6_{i} P<.001\right)$. The contraceptive vital sign increased documentation of use of hor-

\section{Table 1. Baseline Characteristics of Participating General Internal} Medicine Physicians and Visits

\begin{tabular}{|c|c|c|c|}
\hline Characteristic & Control & Intervention & $P$ Value $^{a}$ \\
\hline Physicians & $n=27$ & $n=26$ & - \\
\hline Female, No. (\%) & $17(63)$ & $14(54)$ & .50 \\
\hline Resident, No. (\%) & $11(41)$ & $13(50)$ & .50 \\
\hline Study visits ${ }^{b}$ per physician, median (IQR), No. & $65(126)$ & $22(68)$ & .13 \\
\hline $\begin{array}{l}\text { Study patients }{ }^{b} \text { per physician, median (IQR) } \\
\text { No. }\end{array}$ & $52(88)$ & $14.5(38)$ & .10 \\
\hline Visits with study physicians & $\mathrm{n}=1,705$ & $\mathrm{n}=773$ & - \\
\hline Patient age, mean (SD), y & $32.6(8.2)$ & $34.7(8.4)$ & .82 \\
\hline$\geq 30$ years of age, No. (\%) & $999(58.6)$ & $545(70.5)$ & .74 \\
\hline Married,No. (\%)c & $546(33.2)$ & $261(34.1)$ & .83 \\
\hline White, No. (\%) & $1,300(76.2)$ & $555(71.8)$ & .86 \\
\hline Visit with patient's usual physician, No. (\%) & $1,284(75.3)$ & $571(73.9)$ & .90 \\
\hline New patient visit, No. (\%) & $35(2.1)$ & $22(2.8)$ & .81 \\
\hline Visit with a female doctor, No. (\%) & $1,389(81.5)$ & $404(52.3)$ & .67 \\
\hline Visit with a resident, No. (\%) & $65(3.8)$ & $51(6.6)$ & .79 \\
\hline $\begin{array}{l}\text { Involved prescription of a potential teratogen, } \\
\text { No. (\%) }\end{array}$ & $313(18.4)$ & $104(13.5)$ & .08 \\
\hline \multicolumn{4}{|l|}{$\mathrm{IQR}=$ interquartile range. } \\
\hline \multicolumn{4}{|c|}{$\begin{array}{l}\text { a } P \text { values for comparison of physician characteristics are from } \chi^{2} \text { and Wilcoxon rank sum tests. } P \text { values for } \\
\text { comparison of visit characteristics are from unadjusted logistic models including physician and patient as } \\
\text { crossed random effects. }\end{array}$} \\
\hline
\end{tabular}

Table 2. Responses to the Contraceptive Vital Sign Questions

\begin{tabular}{|c|c|c|}
\hline Question and Responses & $\begin{array}{l}\text { General Internal } \\
\text { Medicine Practice, } \\
\text { No. (\%) }\end{array}$ & $\begin{array}{c}\text { Family Medicine } \\
\text { Practice, } \\
\text { No. }(\%)\end{array}$ \\
\hline $\begin{array}{l}\text { Are you currently pregnant or trying } \\
\text { to become pregnant? }\end{array}$ & $n=758$ & $\mathrm{n}=941$ \\
\hline No, and not trying to get pregnant & $699(92.2)$ & $849(90.2)$ \\
\hline Not trying, but wouldn't mind being pregnant & $47(6.2)$ & $34(3.6)$ \\
\hline Trying to get pregnant & $2(0.3)$ & $28(3.0)$ \\
\hline Currently pregnant & $3(0.4)$ & $20(2.1)$ \\
\hline Prefer not to answer & $5(0.7)$ & $10(1.1)$ \\
\hline $\begin{array}{l}\text { There are many ways that people try } \\
\text { to avoid becoming pregnant. Which } \\
\text { are you using? }\end{array}$ & $\mathrm{n}=746^{\mathrm{a}}$ & $\mathrm{n}=849^{b}$ \\
\hline Using contraception & $392(52.5)$ & $567(66.8)$ \\
\hline Barrier or behavioral contraception & $139(18.6)$ & $171(20.1)$ \\
\hline Pill, patch, or ring & $169(22.7)$ & $296(34.9)$ \\
\hline Intrauterine or subdermal contraception & $52(7.0)$ & $21(2.5)$ \\
\hline Partner's vasectomy & $32(4.3)$ & $79(9.3)$ \\
\hline Sexually active without contraception & $87(11.7)$ & $119(14.0)$ \\
\hline $\begin{array}{l}\text { Not sexually active with man in the last } \\
3 \text { months }\end{array}$ & $220(29.5)$ & $84(9.9)$ \\
\hline Prefer not to answer & $47(6.3)$ & $79(9.3)$ \\
\hline
\end{tabular}


Figure 2. Change in proportion of visits with medical record documentation of contraception before and after introduction of the contraceptive vital sign.

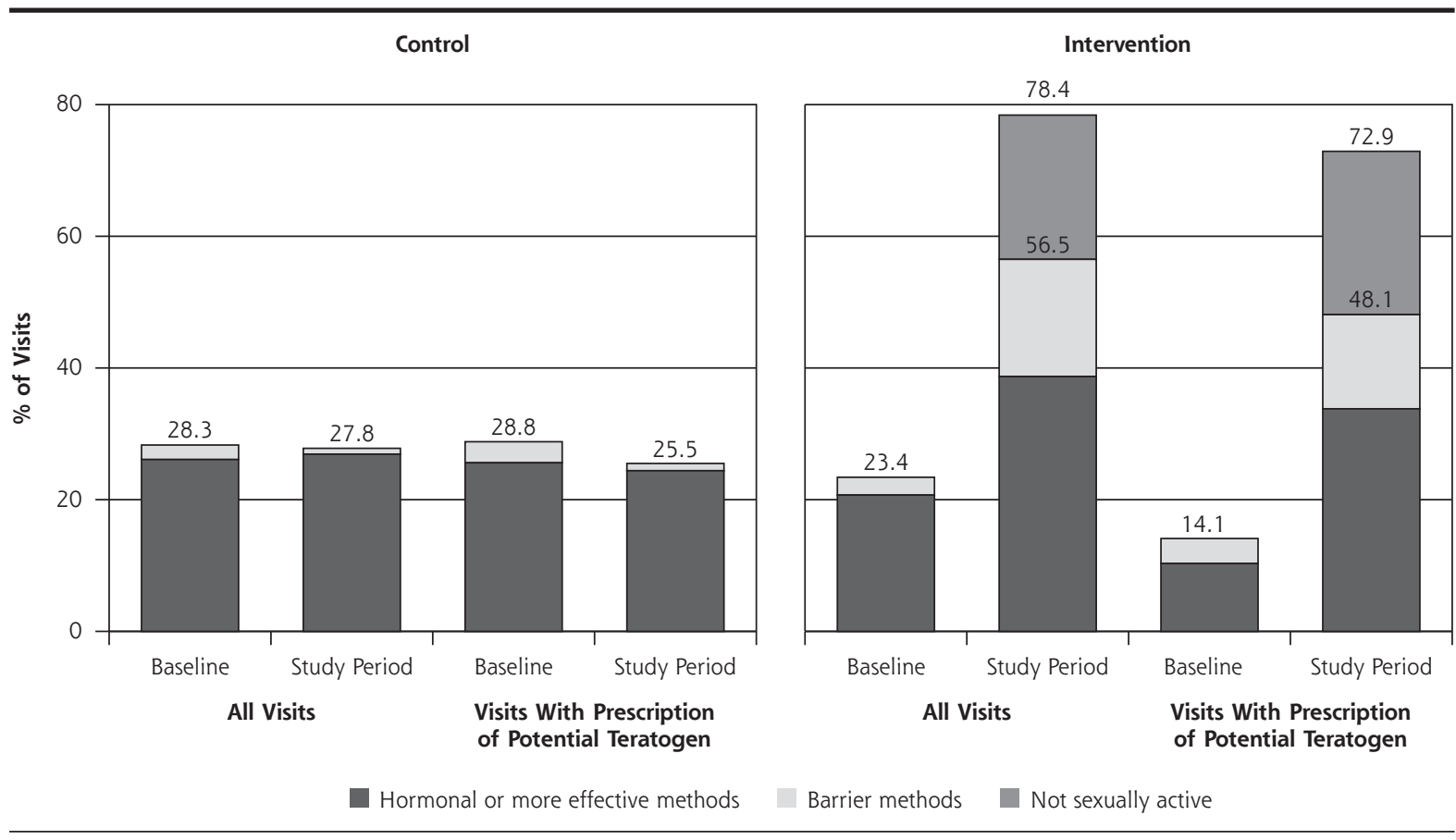

Notes: Contraceptive vital sign data and electronic health record data were collected between October 1, 2008, and April 14, 2010. Baseline electronic health record data from the 17 months before introduction of the contraceptive vital sign were used for comparison. In generalized linear mixed-effects models, there was a greater increase in documentation of contraception in the intervention group compared with the control group, both for all visits $(P<.001)$ and visits that involved prescription of potentially teratogenic medications $(P<.001)$. Hormonal or more effective methods = pill, patch, ring, injection, intrauterine devices, subdermal contraceptive implants, and vasectomies. Women who had been sterilized were excluded. Not sexually active $=$ women who reported no sex with a man in past 3 months.

which women had not been sexually active in the past 3 months.

At baseline, $14 \%$ of intervention visits and $18 \%$ of control visits included prescriptions for potentially teratogenic medications $(P<.01)$. After introduction of the contraceptive vital sign, there was little change in the rate of teratogenic prescriptions for either group: $+2.6(95 \% \mathrm{CI},-0.8$ to +6.0$)$ adjusted percentage points in the intervention group vs $-0.4(95 \% \mathrm{CI},-3.0$ to $+2.0)$ in the control group $(P=.1)$. At baseline, family planning services were provided at $9 \%$ of intervention visits and $11 \%$ of control visits $(P=1.0)$. After introduction of the contraceptive vital sign, intervention physicians were not significantly more likely to provide new family planning services than control physicians: +0.3 (95\% CI, -2.8 to 3.3$)$ vs $-1.4(95 \% \mathrm{CI},-3.3$ to 0.4$)$ adjusted percentage points $(P=.3)$. The ICC was 0.01 (95\% CI, -0.01 to 0.03 ) for potentially teratogenic prescriptions and 0.03 (95\% CI, 0.002 to 0.048$)$ for family planning services.

Among the visits with potentially teratogenic prescriptions, at baseline, family planning services were provided at $7 \%$ of intervention visits and $12 \%$ of control visits $(P=.7)$. Again, however, there was only minimal increase in provision of new family planning services by intervention physicians: +3.3 (95\% CI, -5.4 to 12.0) adjusted percentage points in the intervention group vs -1.7 (95\% CI, -6.6 to 3.3$)$ in the control group $(P=.3)$. The ICC for family planning services was less than $0.001(95 \% \mathrm{CI},-0.02$ to 0.02$)$ in this subgroup of visits with prescription of potential teratogens.

Of the 133 visits to intervention physicians during the study period that involved prescription of a potential teratogen, $17 \%$ still had no documentation of the patient's contraceptive status (either because they did not complete the contraceptive vital sign questions [9\%] or responded "prefer not to answer" [8\%]), and $11 \%$ had documentation of nonuse of contraception. Of the 14 visits with documentation of contraceptive nonuse, only $1(7 \%)$ received a referral for family planning services; none received pregnancy testing or a new contraceptive prescription.

At the community-based family practice, new family planning services were provided during $9 \%$ of visits, whether or not contraceptive vital sign data were collected. Visits with contraceptive vital sign data were significantly less likely to include prescriptions for teratogenic medications: $-3.8(95 \% \mathrm{CI},-7.1$ to -0.3$)$ adjusted percentage points $(P=.03)$. But visits with potential teratogens were not more likely to include 
provision of new family planning services when contraceptive vital sign data were collected: $-3.2(95 \% \mathrm{CI}$, -6.3 to -0.1$)$ adjusted percentage points $(P=.04)$

\section{DISCUSSION}

This study found that routine collection of contraceptive vital sign data was acceptable to the large majority of women served by 2 primary care practices and significantly improved documentation of contraception. The intervention had minimal impact on documented provision of family planning services, however, and a substantial number of patients prescribed potentially teratogenic medications were found to remain at risk for unintended pregnancy. Although the intervention was designed to limit primary care physicians' liability when a potential teratogen was prescribed, it may have inadvertently increased liability when contraceptive nonuse was explicitly documented alongside a potentially teratogenic prescription.

This randomized intervention took place in an academic general internal medicine practice that trains residents and has established an efficient electronic intake system. These findings thus may not be generalizable to all primary care settings; however, results from our pilot study in a community-based family practice were similar. Compared with general internists, family physicians see a larger proportion of female patients of reproductive age, ${ }^{2,13}$ receive more training in reproductive health, and report being more comfortable providing routine gynecologic care and initiating contraception. ${ }^{14}$ Nationally, family physicians tend to provide contraceptive counseling more frequently than general internists $(10.2 \%$ of visits to former vs $6.4 \%$ of visits to latter). ${ }^{2}$ Family physicians are somewhat less likely to prescribe potentially teratogenic medications $(6.4 \%$ vs $8.0 \%$ of visits). ${ }^{2}$ Nonetheless, provision of appropriate counseling when teratogenic medications are prescribed remains a considerable challenge for both family physicians and general internists, as primary care physicians generally have lower levels of contraceptive knowledge than gynecologists. ${ }^{15-17}$

Recently an extensive educational intervention was shown to increase family practice residents' documentation of contraceptive counseling when prescribing teratogenic medications from $20 \%$ to $37 \% .{ }^{18}$ In a similar study, documentation of contraceptive counseling rose from $46 \%$ to $80 \%$ of family practice visits involving a teratogenic medication, ${ }^{19}$ although the authors were unable to be certain that this was the result of their intervention as documentation of contraception had been rising before the intervention and there was no control group. A major strength of our study is its randomized controlled design. Also, although it took place in a practice that uses an electronic intake system, contraceptive vital sign data could easily be assessed by nursing staff or using a paper intake system.

As primary care physicians frequently discuss contraception during annual preventive visits, it is unfortunate that a programming error excluded these visits from our study, and the final sample size was smaller than intended. With only 110 visits that included a warning message, we had limited power to detect significant improvement in physician behaviors. Although the majority of clinicians in the study clinic felt that women who had not been sexually active in the last 3 months were at low risk for pregnancy, some of these women may have resumed sexual activity after filling a teratogenic prescription and might have benefited from contraceptive counseling. As more than one-third of women see both a primary care physician and gynecologist, ${ }^{20}$ we would have liked to ask women if they wanted to discuss birth control with their primary care physician; however, concerns about the length of the intake questionnaire precluded the addition of more than the 2 questions we studied at this time. Additionally, as primary care physicians have little incentive to code for provision of counseling services and we did not review physician notes from these visits, physicians likely provided preconception and contraceptive counseling more often than is reflected in the EHR data. A study by Gilchrist et $\mathrm{al}^{21}$ suggests that $71 \%$ of visits wherein contraceptive counseling occurs lack documentation of this counseling. As some physicians who felt pressured for time may have planned to provide such counseling at a follow-up visit, the fact that we examined data from only 1 visit may underestimate the intervention's true effect. Additionally, we have no way of verifying that physicians in either the general medicine or family practice reviewed the contraceptive vital sign data that was provided, as they were not instructed to look for it or to document counseling in the EHR.

In conclusion, a contraceptive vital sign improves primary care documentation of pregnancy intentions and contraception in a manner that is acceptable to patients. Prior research indicates that women prescribed teratogenic medications prefer to hear about the risks of these medications directly from their prescribing physician, ${ }^{22}$ and provision of contraceptive counseling in primary care has been associated with increased likelihood of contraceptive use. ${ }^{23}$ Ongoing efforts are needed, however, to ensure that primary care patients receive preconception counseling and family planning services, and that the provision of these services is documented in the EHR, particularly when potentially teratogenic medications are prescribed.

To read or post commentaries in response to this article, see it online at http://www.annfammed.org/content/10/6/516. 
Key words: preconception care; contraception; primary healthcare; teratogens; health services research

Submitted August 24, 2011; submitted, revised, December 21, 2011; accepted February 22, 2012.

Funding support: This study was funded by AHRQ R18HS017093 and NICHD K23HD051585. Ms Williams was supported by a grant from the Doris Duke Charitable Foundation to the University of Pittsburgh.

Previous presentation: A poster describing a preliminary analysis of this data was presented at the Reproductive Health Conference in Atlanta, GA, on September 22-25, 2010.

Trial Registration: ClinicalTrials.gov Identifier: NCT00766207

\section{References}

1. Finer LB, Henshaw SK. Disparities in rates of unintended pregnancy in the United States, 1994 and 2001. Perspect Sex Reprod Health. 2006;38(2):90-96.

2. Schwarz EB, Maselli J, Norton M, Gonzales R. Prescription of teratogenic medications in United States ambulatory practices. Am J Med. 2005;118(11):1240-1249.

3. Andrade SE, Gurwitz JH, Davis RL, et al. Prescription drug use in pregnancy. Am J Obstet Gynecol. 2004;191(2):398-407.

4. Schwarz EB, Postlethwaite DA, Hung YY, Armstrong MA. Documentation of contraception and pregnancy when prescribing potentially teratogenic medications for reproductive-age women. Ann Intern Med. 2007;147(6):370-376.

5. Schwarz EB, Santucci A, Borrero S, Akers AY, Nikolajski C, Gold MA. Perspectives of primary care clinicians on teratogenic risk counseling. Birth Defects Res A Clin Mol Teratol. 2009;85(10):858-863.

6. Eisenberg DL, Stika C, Desai A, Baker D, Yost KJ. Providing contraception for women taking potentially teratogenic medications: a survey of internal medicine physicians' knowledge, attitudes and barriers. J Gen Intern Med. 2010;25(4):291-297.

7. American Pain Society Quality of Care Committee. Quality improvement guidelines for the treatment of acute pain and cancer pain. JAMA. 1995;274(23):1874-1880.

8. Veterans Health Administration. Pain as the 5th Vital Sign Toolkit. Revised edition. October 2000. http://www.va.gov/PAINMANAGEMENT/docs/TOOLKIT.pdf. Accessed Dec 16, 2011.

9. Hess R, Matthews K, McNeil M, Chang CH, Kapoor W, Bryce C. Health services research in the privacy age. J Gen Intern Med. 2005; 20(11):1045-1049.
10. Hess R, Santucci A, McTigue K, Fischer G, Kapoor W. Patient difficulty using tablet computers to screen in primary care. J Gen Intern Med. 2008;23(4):476-480.

11. Murray DM. Design and Analysis of Group-Randomized Trials. New York, NY: Oxford University Press; 1998.

12. Raudenbush SW, Bryk AS. Hierarchal Linear Models: Applications and Data Analysis Methods. Thousand Oaks, CA: Sage Publications; 2002.

13. National Center for Health Statistics. Table 92: Visits to primary care generalist and specialist physicians, by selected characteristics and type of physician: United States, selected years 1980- 2008. In Health, United States, 2010. http://www.cdc.gov/nchs/data/hus/ hus10.pdf. Accessed Dec 16, 2011.

14. Dixon JG, Bognar BA, Keyserling TC, et al. Teaching women's health skills: confidence, attitudes and practice patterns of academic generalist physician. J Gen Intern Med. 2003;18(6):411-418.

15. Schreiber CA, Harwood BJ, Switzer GE, Creinin MD, Reeves MF, Ness RB. Training and attitudes about contraceptive management across primary care specialties: a survey of graduating residents. Contraception. 2006;73(6):618-622.

16. Dehlendorf C, Levy K, Ruskin R, Steinauer J. Health care providers' knowledge about contraceptive evidence: a barrier to quality family planning care? Contraception. 2010;81(4):292-298.

17. Spagnoletti CL, Rubio DM, McNeil MA. Internal medicine residents' preparedness to care for reproductive-age and pregnant women. Teach Learn Med. 2007;19(3):257-263.

18. Morrical-Kline KA, Walton AM, Guildenbecher TM. Teratogen use in women of childbearing potential: an intervention study. J Am Board Fam Med. 2011;24(3):262-271.

19. Fritsche MD, Ables $A Z$, Bendyk H. Opportunities missed: improving the rate of contraceptive counseling or provision when prescribing reproductive-aged women potentially teratogenic medications in a family medicine resident clinic. Contraception. 2011;84(4):372-376.

20. Weisman CS. Women's use of health care. In: Falik M, Collins K, eds. Women's Health: the Commonwealth Fund Survey of Women's Health. Baltimore, MD: Johns Hopkins University Press; 1996.

21. Gilchrist VJ, Stange KC, Flocke SA, McCord G, Bourguet CC. A comparison of the National Ambulatory Medical Care Survey (NAMCS) measurement approach with direct observation of outpatient visits. Med Care. 2004;42(3):276-280.

22. Santucci AK, Gold MA, Akers AY, Borrero S, Schwarz EB. Women's perspectives on counseling about risks for medication-induced birth defects. Birth Defects Res A Clin Mol Teratol. 2010;88(1):64-69.

23. Lee JK, Parisi SM, Akers AY, Borrero S, Schwarz EB. The impact of contraceptive counseling in primary care on contraceptive use. J Gen Intern Med. 2011;26(7):731-736. 